\title{
Profile of tofacitinib citrate and its potential in the treatment of moderate-to-severe chronic plaque psoriasis
}

This article was published in the following Dove Press journal:

Drug Design, Development and Therapy

29 January 2016

Number of times this article has been viewed

\author{
V Di Lernia' \\ F Bardazzi² \\ 'Dermatology Unit, Arcispedale Santa \\ Maria Nuova IRCCS, Reggio Emilia, \\ ${ }^{2}$ Division of Dermatology, Department \\ of Experimental, Diagnostic and \\ Specialty Medicine, University of \\ Bologna, Bologna, Italy
}

\begin{abstract}
The outlook for patients with psoriasis has improved significantly over the last 10 years with the introduction of targeted therapies. Cytokines exert their effects by activating intracellular signaling and transcription pathways, among which there are Janus kinases (JAKs) and signal transducers and activators of transcription (STAT) pathways. JAKs are intracellular second messengers that are crucial for transmitting extracellular cytokine signals to the cell. JAK inhibition interrupts intracellular signaling and can suppress immune cell activation and inflammation in T-cell-mediated disorders, such as psoriasis. Consequently, JAKs are the subject of intensive research activity, since they represent possible therapeutic targets. Tofacitinib is an orally available compound belonging to a novel category of nonbiologic drugs, the "JAK inhibitors", which target JAKs. Recently, oral and topical formulations of tofacitinib have been demonstrated to be safe and effective for the treatment of plaque psoriasis in randomized clinical trials. In particular, a $10 \mathrm{mg}$ bid dose of tofacitinib was shown to be noninferior to etanercept $50 \mathrm{mg}$ subcutaneously twice weekly. Questions remain unresolved regarding the safety risk beyond the $5 \mathrm{mg}$ bid dose. This review, assessing the available scientific literature, focuses on the profile of tofacitinib, as investigational compound in the treatment of plaque psoriasis. An overview of the efficacy and safety data from randomized clinical trials is provided. In addition, the authors highlight future potential applications of tofacitinib in other skin diseases, in particular alopecia areata and vitiligo.
\end{abstract}

Keywords: treatment, therapy, systemic, JAKs, vitiligo, alopecia

\section{Introduction}

Psoriasis is a highly heritable common, chronic inflammatory skin disease with a high familial recurrence risk. ${ }^{1}$ It affects $1 \%-3 \%$ of the world's population. Chronic plaque psoriasis is the most common form of the disease that is clinically characterized by well-delineated red and scaly plaques.

Psoriasis has a multifactorial origin. The central processes underlying its pathogenesis are inflammation and epidermal hyperproliferation, which are believed to be consequences of a dysregulated interaction of the innate and adaptive immune system in the context of skin epithelium and connective tissue. ${ }^{2}$ The course of psoriasis in any individual patient is variable and difficult to predict with accuracy. ${ }^{3}$ In patients with early onset, the disease often follows an irregular course with tendency to become severe and extensive. ${ }^{4}$ Psoriasis is a major risk factor for the development of psoriatic arthritis, a heterogeneous inflammatory arthritis with a variable clinical course. ${ }^{5}$ It belongs to the spondyloarthritis group and affects primarily the peripheral joints, the spine, and the entheses. Joint disease is characterized by systemic inflammation and
Correspondence: V Di Lernia

Dermatology Unit, Arcispedale Santa Maria Nuova IRCCS, Viale Risorgimento 80, Reggio Emilia 42I23, Italy Email vito.dilernia@asmn.re.it (c) (i) (5) 2016 Di Lernia and Bardazzi. This work is published and licensed by Dove Medical Press Limited. The full terms of this license are available at https://www.dovepress.com/terms.php

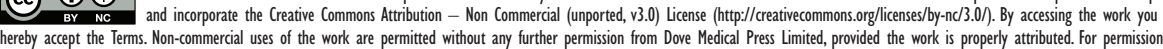
hereby accept he This. Non-commercial uses of the work are permitted without any further permission from Dove 
extensive synovitis, resulting in erosions of articular cartilage leading to joint destruction. In patients with psoriasis, associated comorbidities may occur more frequently than expected. Psoriasis is an independent risk factor for cardiovascular ${ }^{6-8}$ and metabolic syndromes. ${ }^{9,10}$ The definition of psoriasis severity helps to classify treatment. Moderate-to-severe psoriasis is defined if the body surface involvement is $>10 \%$ and/ or if Psoriasis Area Severity Index (PASI) is $>10$, although particular clinical situations may change mild psoriasis to moderate-to-severe psoriasis according to involvement of visible areas or marked nail involvement. ${ }^{11}$

Various conventional and biologic systemic agents may be chosen to treat patients with moderate-to-severe psoriasis. In the last decade, several cellular and molecular mediators in psoriasis have been identified. They included first tumor necrosis factor (TNF)-alpha, then interleukin 12 (IL-12) and IL-23 and more recently IL-17. Such cytokines are pivotal in the disease process. Limiting the interaction of specific cytokines with their specific receptors has been successfully exploited for therapeutic purposes through the development and characterization of monoclonal antibodies or soluble receptors. Consequently, biologic therapies targeting specific immune pathways have emerged for the treatment of moderate-to-severe plaque psoriasis. ${ }^{12}$ Despite the availability of a broad spectrum of treatments, additional therapeutic options with distinctive mechanism of action may be advantageous for the management of the disease. ${ }^{13,14}$ Indeed, the overall efficacy of TNF-alpha inhibitors diminishes with time. ${ }^{15-17}$ The body mass index affects the long-term survival rate of TNF-alpha blockers in psoriatic patients. ${ }^{18}$ Loss of efficacy can also occur over time with the use of biologics due to their potential immunogenicity. ${ }^{19}$ Otherwise the injection schedule of treatment has been cited by patients as reasons contributing to treatment discontinuation. ${ }^{20}$

Recently, attention has been addressed to new compounds, namely small molecular weight inhibitors. Such molecules target intracellular signaling pathways. Small molecular weight inhibitors have some interesting features in that, as synthetic compounds, they are relatively inexpensive to produce and can be administered orally. ${ }^{21}$

The purpose of this review is to present updated data available on the pharmacology, efficacy, safety, and tolerability of tofacitinib, an orally available compound belonging to a novel class of drugs, the Janus kinase (JAK) inhibitors, in the treatment of plaque psoriasis. After introducing the mechanism of action, an overview of the efficacy and safety results from the randomized clinical trials of tofacitinib in patients affected by plaque psoriasis is presented. Further, future research directions in other skin diseases are highlighted.

\section{Protein kinase family and Janus family of tyrosine kinases}

Protein kinases are the second largest human protein family. They are involved in protein phosphorylation, a fundamental component of cell signaling. Aberrant kinase activity is linked either directly or indirectly to a consistent number of diseases. Therefore, kinases are viewed as potential drug targets. ${ }^{22}$

A family of kinases are the JAKs, of which there are four members, JAK1, JAK2, JAK3, and tyrosine kinase 2 (TYK2). ${ }^{23} \mathrm{JAKs}$ are intracellular second messengers that are crucial for transmitting extracellular cytokine signals to the cell. They regulate cellular processes involved in cell growth, differentiation, and transformation through their association with cytokine receptors. The function of JAKs was discovered by the generation of cell lines resistant to the effects of interferons, whose defects could be balanced by expression of different JAKs. ${ }^{24-26}$ A large number of cytokines activate the JAK/signal transducers and activators of transcription (STAT) pathway. ${ }^{27} \mathrm{JAK} 1$ binds with $\gamma$-chain cytokines (IL-6, IL-10, IL-13, IL-22, granulocyte colony-stimulating factor, interferons). JAK3 binds to the common IL-2R $\gamma$ chain of the type I cytokine receptor family (IL-2, IL-4, IL-7, IL-9, IL-15, IL-21). All these cytokines are crucial for lymphocyte activation, proliferation, and function. Inhibition of their signaling may thus result in modulation of multiple aspects of the immune response. ${ }^{28-30}$ JAK2 is associated with hematopoietic growth factor receptors. ${ }^{29}$ JAK1, JAK2, and TYK2 are expressed ubiquitously in mammals. The expression of JAK3 is limited to hematopoietically derived cells. ${ }^{31}$ The deficiency or dysfunction of JAK3 correlates with impairment of lymphocyte development and function and leads to severe combined immunodeficiency. ${ }^{32}$

JAKs are critical for both innate and adaptive immunity. The noticeable upregulation of JAK/STAT pathways in psoriasis and the recognition of multiple key mediators in psoriasis pathogenesis that signal through JAK/STAT pathways led to consider JAK proteins as potential therapeutic targets for psoriasis treatment as well. ${ }^{33}$

\section{JAK inhibitors and tofacitinib: mechanism of action}

As stated earlier, psoriasis is characterized by activation of several different types of immune cells that infiltrate the skin, stimulating keratinocytes to proliferate and produce proinflammatory cytokines. Effective activation of immune 
cells needs integration of external stimuli through their surface receptors. The attachment between the receptor and its ligand results in activation of intracellular kinases. ${ }^{34}$ Such enzymes phosphorylate downstream molecules, propagating a cascade that leads to transcription of genes coding effector molecules. Therefore, JAK inhibition can suppress immune cell activation and inflammation in psoriasis as well ${ }^{35}$ (Figure 1).

Tofacitinib, an orally available compound, is the first member of a novel class of medications, the JAK inhibitors. It inhibits phosphorylation of JAK1 and JAK3, IL-6-driven phosphorylation of STAT1 and STAT3, and STAT5. ${ }^{30,36}$ However, it is currently categorized as a pan-JAK inhibitor preferentially inhibiting JAK1 and JAK3 and, to a lesser extent, JAK2 with minimum effect on TYK2. ${ }^{29,37,38}$ The primary targets of tofacitinib are dendritic cells, CD4(+) T-cells such as Th1 and Th17, and activated B-cells that lead to multicytokine targeting. ${ }^{39}$ Tofacitinib is able to inhibit antigen-presentation and T-cell stimulatory capacity of dendritic cells by inhibiting type I interferon-mediated signaling. ${ }^{39}$ Subsequently, the expression of costimulatory molecules such as CD80 and CD86 is reduced as well. ${ }^{39}$ Tofacitinib is able to inhibit differentiation and antibody production of $\mathrm{B}$-cells, ${ }^{39}$ whereas it maintains B-cell regulatory function. Tofacitinib limits the production of IL-17A, IL-17F, and IL-22, the expression of the IL-23R as well as differentiation of Th1 cells. ${ }^{29,36}$ Since IL-23 is one of the key cytokines that shapes the development and function of Th17 cells, by suppressing the IL-23 receptor expression, the Th17 cell differentiation is consequently reduced..$^{36,39-42}$

\section{Oral tofacitinib: clinical trials in psoriasis}

The efficacy and safety of tofacitinib was investigated in placebo-controlled and head-to-head randomized clinical trials in patients with moderate-to-severe chronic plaque psoriasis. ${ }^{43,44}$ In two similarly designed Phase III placebocontrolled studies, higher response rates were seen with the $10 \mathrm{mg}$ bid dosage regimen of tofacitinib. ${ }^{43}$ After 16 weeks, $75 \%$ reduction in PASI 75 rates were $39.9 \%$ and $59.2 \%$, respectively, for tofacitinib 5 and $10 \mathrm{mg}$ bid (Opt Pivotal 1 study), $46.0 \%$, and $59.6 \%$, respectively, for tofacitinib 5 and $10 \mathrm{mg}$ bid (Opt Pivotal 2 study) (Table 1).

In a head-to-head randomized clinical trial versus etanercept, tofacitinib was noninferior to etanercept only with the $10 \mathrm{mg}$ bid dose. ${ }^{44}$ The Phase III, double-dummy, placebo-controlled, 12-week trial, comparing two tofacitinib doses with high-dose etanercept or placebo enrolled 1,106 psoriatic patients with a PASI score of 12 or higher and a Physician Global Assessment (PGA) of moderate or severe who had failed to respond to, had a contraindication

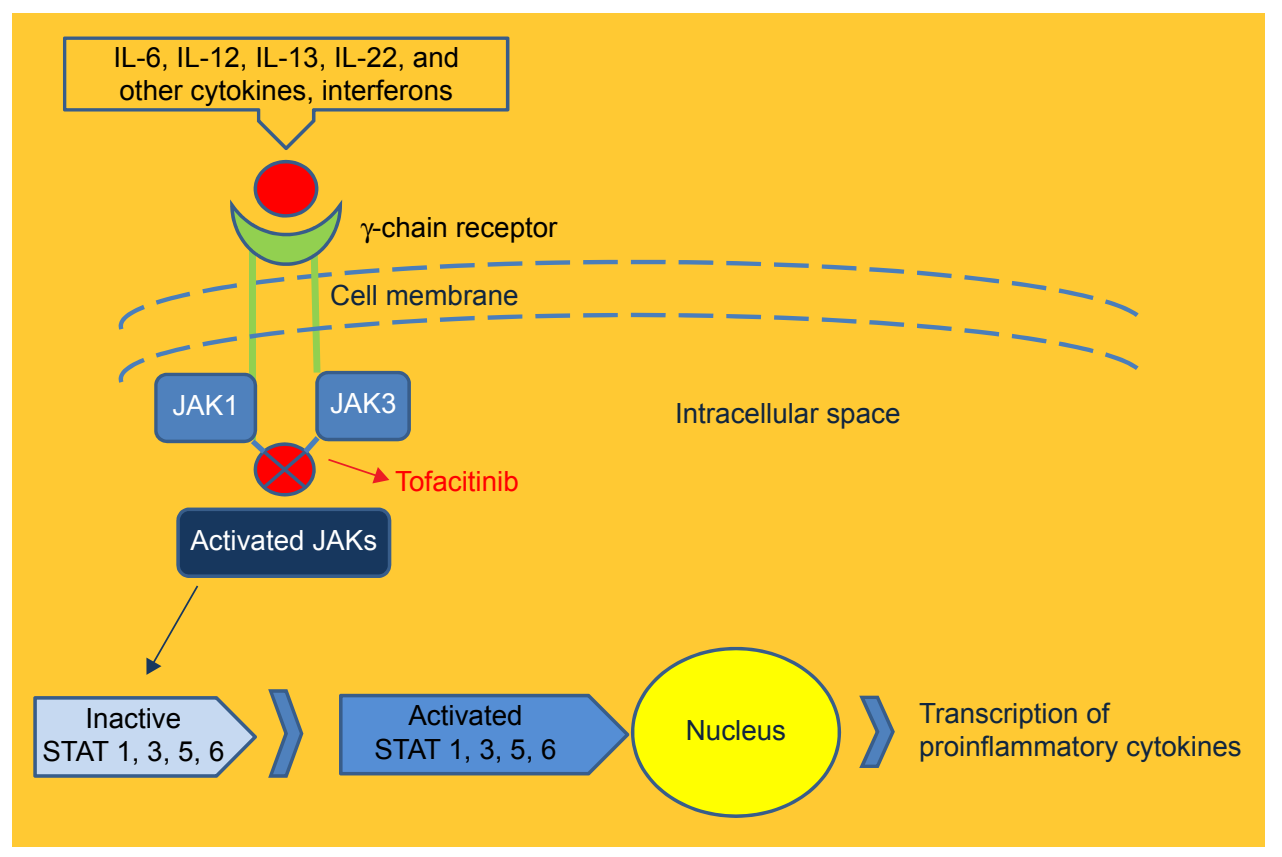

Figure I Binding of cytokines to the receptor in turn activates an intracellular signaling cascade via JAKs with subsequent phosphorylation of STATs.

Notes: JAKs are thought to be activated through phosphorylation. Tofacitinib inhibits the phosphorylation and activation of JAKs. JAKs cannot phosphorylate the cytokine receptors. Consequently the receptors cannot dock STATS. These latter are not phosphorylated and activated. Therefore, they cannot translocate to the nucleus. Gene transcription and cytokine production are inhibited.

Abbreviations: IL, interleukin; JAK, Janus kinase; STAT, signal transducers and activators of transcription. 
Table I Results summary for primary outcomes of Phase III published trials of tofacitinib in moderate-to-severe psoriasis

\begin{tabular}{|c|c|c|}
\hline $\begin{array}{l}\text { Tofacitinib } \\
\text { (trial) }\end{array}$ & $\begin{array}{l}\text { Outcome } \\
\text { PASI } 75 \text { (\%) }\end{array}$ & $\begin{array}{l}\text { Outcome } \\
\text { PGA (\%) }\end{array}$ \\
\hline $5 \mathrm{mg}$ bid (Opt Pivotal I) ${ }^{43}$ & 39.9 & 41.9 \\
\hline $5 \mathrm{mg}$ bid (Opt Pivotal 2$)^{43}$ & 46.0 & 46.0 \\
\hline $5 \mathrm{mg}$ bid (Opt Compare) $)^{44}$ & 39.5 & 47.1 \\
\hline 10 mg bid (Opt Pivotal I) & 59.2 & 59.2 \\
\hline 10 mg bid (Opt Pivotal 2) ${ }^{43}$ & 59.6 & 59.1 \\
\hline $10 \mathrm{mg}$ bid (Opt Compare) ${ }^{44}$ & 63.6 & 68.2 \\
\hline
\end{tabular}

Note: Data from Papp et $\mathrm{al}^{43}$ and Bachelez et al. ${ }^{44}$

Abbreviations: PASI, Psoriasis Area Severity Index; PGA, Physician Global Assessment.

to, or were intolerant to at least one conventional systemic therapy. Eligible patients were randomly assigned to receive tofacitinib 5 or $10 \mathrm{mg}$ bid, etanercept $50 \mathrm{mg}$ subcutaneously twice weekly or placebo. The co-primary endpoints were the proportion of patients at week 12 with at least a $75 \%$ reduction in the PASI 75 response from baseline and the proportion of patients achieving a PGA score of "clear" or "almost clear". At week 12, PASI 75 responses were recorded in $63.6 \%$ of patients in the tofacitinib $10 \mathrm{mg}$ group, compared with $39.5 \%$ in the tofacitinib $5 \mathrm{mg}$ group, $58.8 \%$ in the etanercept group, and $5.6 \%$ in the placebo group. A total of $68.2 \%$ of patients treated with tofacitinib $10 \mathrm{mg}$ achieved the other primary endpoint of PGA score of clear or almost clear by week 12 , compared with $66.3 \%$ of who received etanercept. Patients treated with the lower $(5 \mathrm{mg})$ dose of tofacitinib had an inferior response, since $47.1 \%$ achieved clear or almost clear skin, compared with $15 \%$ of the placebo group. For both co-primary endpoints, the $10 \mathrm{mg}$ dose of tofacitinib was noninferior to etanercept and superior to placebo, whereas the $5 \mathrm{mg}$ tofacitinib dose did not meet the noninferiority criteria versus etanercept, but met the superiority criteria versus placebo (Table 1). ${ }^{44}$

Another Phase III trial investigated outcomes following tofacitinib withdrawal with outcomes of continuation in patients who had achieved both $\geq 75 \%$ reduction in PASI score from baseline and PGA response. After initial treatment of 24-week duration, 33.5\% and 55.2\% achieved both PASI 75 and PGA responses with tofacitinib 5 and $10 \mathrm{mg}$ bid, respectively. Following tofacitinib withdrawal, patients who received continuous treatment maintained a response more effectively when compared with placebo recipients. Of those patients who relapsed, up to $60 \%$ recaptured a response with tofacitinib. ${ }^{45}$ Best response rate with oral tofacitinib were seen with the $10 \mathrm{mg}$ bid dose in this study as well.

\section{Safety}

By blocking JAK signaling, tofacitinib modulates the immune response via downregulation of several cytokines, including IL-2, IL-4, IL-7, IL-9, IL-15, and IL-21, which are integral to lymphocyte development and function. ${ }^{46}$ Safety considerations must be considered especially beyond the 5 mg bid dose. ${ }^{43}$ The risk of tuberculosis and other opportunistic infections may potentially be elevated in tofacitinib-treated patients. Overall tuberculosis cases occurred in patients using the higher dose of $10 \mathrm{mg}$ bid. ${ }^{47}$ Decrease of hemoglobin, neutrophil, and lymphocyte counts, and increase from baseline in creatinine and alanine aminotransferase may occur, as well as dyslipidemia. Changes of mean serum creatinine levels do not appear to be associated with acute renal failure or progressive worsening of renal function. ${ }^{48}$ Dyslipidemia is dose-related and affects total cholesterol, high-density lipoprotein cholesterol, and low-density lipoprotein cholesterol. Most of these abnormalities are transient and reversible either spontaneously or with discontinuation of the study drug. ${ }^{43,48}$

In Phase III clinical trials, the rate of adverse events were similar across the different groups investigated ${ }^{43,44}$ and a safety profile similar to that of etanercept was observed in the short-term. ${ }^{44}$ About infectious events, upper respiratory tract infections, sinusitis, nasopharyngitis, and herpes zoster were reported in patients treated with tofacitinib. ${ }^{43}$ Information about long-term safety of tofacitinib may be indirectly extrapolated from long-term extension studies and systematic reviews of data of patients with moderate-to-severe active rheumatoid arthritis (RA). ${ }^{48,49}$ A dose-dependency for serious infections was observed. Rates for safety events were consistent with the published rates for TNF inhibitors and other biologic disease-modifying antirheumatic drugs in similar patient populations for mortality, serious infections, including opportunistic infections and tuberculosis, cardiovascular events, and malignancies. ${ }^{48,49}$ Since patient population studied was affected by RA, a potential impact of similarities and differences in the disease state, comorbidities, and treatment paradigms must be taken into account.

\section{Interactions}

Nonrenal elimination accounts for $70 \%$ of the total clearance of tofacitinib. The metabolism is primarily mediated by cytochrome P450 3A4 (CYP3A4) (53\%) with minor contribution by CYP450 2C19 (CYP2C19) (17\%). Therefore, potent inhibitors or inducers of CYP3A4 and CYP2C19 may potentially interact with tofacitinib. Indeed, potent inducers of CYP3A4, such as rifampin, may reduce the clinical efficacy of tofacitinib. Conversely, it is recommended that the dose of tofacitinib should not exceed $5 \mathrm{mg}$ bid in patients who are receiving concomitant potent inhibitors of CYP3A4, such as ketoconazole, or CYP2C19, such as fluconazole. ${ }^{50}$ A study 
by Gupta et $\mathrm{a}^{51}$ did not confirm an inhibitive or inductive effect of tofacitinib on CYP3A4 activity in humans and, in conjunction with in vitro data, supported the conclusion that tofacitinib is unlikely to influence the CYP enzyme system as a whole.

\section{Topical tofaticinib}

Two randomized studies investigated the safety and efficacy of a topical solution of tofacitinib in patients with chronic plaque psoriasis. ${ }^{52,53}$ The first one, a Phase IIa study, met its primary endpoint of efficacy for one of the two ointments containing tofacitinib versus vehicle. ${ }^{52}$ The second one, an intrasubject, left-right, controlled study, enrolled patients who were randomized to $2 \%, 0.2 \%$, or $0.02 \%$ tofacitinib or vehicle solution once or twice daily. ${ }^{53}$ Patients treated one plaque as per their randomization group and used vehicle to treat the contralateral plaque for 4 weeks. The primary efficacy endpoint, consisting of intrasubject, vehicle-adjusted mean percent change from baseline in Target Plaque Severity Score at week 4, was not substantially different from baseline for any treatment group. However, skin biopsy analyses detected tofacitinib in both tofacitinib- and vehicle-treated plaques of some patients, suggesting cross-contamination or solution misapplication. Thus, the authors hypothesized that lack of efficacy with tofacitinib relative to vehicle may be due to the intrasubject study design with unsupervised applications. $^{53}$

\section{Tofacitinib in other diseases: future directions}

Tofacitinib demonstrated superior efficacy to methotrexate and similar efficacy to adalimumab in RA. ${ }^{54,55}$ It gained approval from Food and Drug Administration and regulatory agencies of more than 20 countries, ${ }^{56}$ becoming the first oral JAK inhibitor indicated for treatment of moderateto-severe RA. In contrast, the European Medicine Agency adopted a negative opinion, recommending the refusal of the marketing authorization due to major concerns about the overall safety profile.

Interestingly, anecdotal clinical experiences suggest a potential effectiveness of tofacitinib in other dermatologic diseases. In particular, tofacitinib has shown success in treating alopecia areata and vitiligo. ${ }^{57,58}$ The immune pathways required for autoreactive $\mathrm{T}$-cell activation in alopecia areata are not clearly defined, but the disease is driven by cytotoxic $\mathrm{CD}^{+} \mathrm{NKG}^{-} \mathrm{D}^{+} \mathrm{T}$ lymphocytes and reversed by JAK inhibition. ${ }^{59}$ The inhibition of JAK/STAT signaling promotes hair growth by stimulating the activation and/ or proliferation of hair follicle stem cells. ${ }^{60}$ About vitiligo, interferon (IFN)- $\gamma$-chemokine axis is active in human disease. In particular, a critical role for the IFN- $\gamma$-induced chemokine CXCL10 in both the progression and maintenance of vitiligo has been suggested. ${ }^{61}$ When IFN- $\gamma$ binds to its receptor, it activates the JAK/STAT pathway, which in turn leads to production of CXCL10 in keratinocytes. ${ }^{58}$ Therefore, a direct CXCL10 inhibition or a JAK/STAT pathway inhibition may represent attractive targets for new treatments strategies.

Recently, tofacitinib citrate was given to six consecutive patients with moderate-to-severe atopic dermatitis (AD) who had who failed to gain results with standard treatment. ${ }^{62}$ Response to treatment was assessed using the scoring of $\mathrm{AD}$ index. Decreased body surface area involvement of dermatitis and decreased erythema, edema/papulation, lichenification, and excoriation were observed in all patients. The scoring of AD index decreased by $66.6 \%$ from 36.5 to $12.2(P<0.05)$ during 8-29 weeks of treatment. No adverse events were observed.

\section{Conclusion}

Increased knowledge of the molecular regulatory mechanisms that impact the pathogenesis of psoriasis and other inflammatory diseases has created new chances for the development of targeted drug therapy. The antipsoriatic therapeutic spectrum could be extended in the future to a number of novel oral compounds, the JAK inhibitors. JAKs are intracellular second messengers that are essential for transmitting extracellular cytokine signals to the cell. JAK inhibition can suppress immune cell activation and inflammation. Tofacitinib is a current investigational drug in psoriasis. It is an orally available compound belonging to the group of JAK inhibitors. Oral and topical formulations of tofacitinib have been demonstrated to be safe and effective for the treatment of plaque psoriasis in Phase III clinical trials. If approved for psoriasis treatment by regulatory agencies, it will be interesting to define its place in the treatment algorithm. The available randomized clinical trials in psoriasis place tofacitinib in the same area of etanercept, as second-line agent alongside the TNF-alpha antagonists. The oral delivery route, and economic issues regarding potential better costeffectiveness ratio with respect to biologics and biosimilars, may be tofacitinib strengths. Given the oral administration route, efficacy and safety of tofacitinib could be compared to available conventional antipsoriatic treatments, such as methotrexate or cyclosporine, in randomized clinical trials. Such studies could provide additional information about potential treatment strategies. About safety, questions remain unresolved. The Food and Drug Administration approved only the $5 \mathrm{mg}$ bid dose in RA on the grounds that a higher dose was not considered to have an adequate risk-to-benefit 
ratio. Nevertheless investigational studies in psoriasis showed that $5 \mathrm{mg}$ bid tofacitinib was less effective than etanercept.

Definitely, current studies show no substantial advantages of tofacitinib in terms of efficacy and safety over existing treatments for psoriasis. A Phase III study assessed its noninferiority (rather than superiority) at the higher dose to etanercept. In consideration of the plethora of emerging antipsoriatic drugs recently approved such as secukinumab or apremilast, or in the pipeline, such as anti-IL-23 antibodies, it seems desirable to further explore tofacitinib for promoting hair growth and/or repigmentation. Both alopecia areata and vitiligo are common diseases with big unmet medical needs. Clinical trials with selective JAK inhibitors are required to dissect key pathogenic pathways.

\section{Disclosure}

The authors report no conflicts of interest in this work.

\section{References}

1. Di Lernia V, Ficarelli E, Lallas A, Ricci C. Familial aggregation of moderate to severe plaque psoriasis. Clin Exp Dermatol. 2014;39:801-805.

2. Boehncke WH. Etiology and pathogenesis of psoriasis. Rheum Dis Clin North Am. 2015;41:665-675.

3. Di Lernia V, Ficarelli E. Current therapeutic approaches of psoriasis are affected by age at disease onset. J Dermatolog Treat. 2014;25:15-17.

4. Henseler T, Christophers E. Psoriasis of early and late onset: characterization of two types of psoriasis vulgaris. J Am Acad Dermatol. 1985; $13: 450-456$

5. Gladman DD, Antoni C, Mease P, Clegg DO, Nash P. Psoriatic arthritis: epidemiology, clinical features, course, and outcome. Ann Rheum Dis. 2005;64(Suppl 2):ii14-ii17.

6. Gelfand JM, Neimann AL, Shin DB, Wang X, Margolis DJ, Troxel AB. Risk of myocardial infarction in patients with psoriasis. JAMA. 2006;11;296:1735-1741.

7. Gisondi P, Dalle Vedove C, Girolomoni G. Patients with psoriasis have a higher prevalence of parental cardiovascular disease. Dermatology. 2011;222:330-335.

8. Gisondi P, Targher G, Bardazzi F, et al. Pharmacological treatment of moderate-severe psoriasis in patients with cardio-metabolic comorbidities. G Ital Dermatol Venereol. 2014;149(Suppl 2):7-11.

9. Gisondi P, Tessari G, Conti A, et al. Prevalence of metabolic syndrome in patients with psoriasis: a hospital-based case-control study. $\mathrm{Br}$ J Dermatol. 2007;157:68-73.

10. Ryan C, Kirby B. Psoriasis is a systemic disease with multiple cardiovascular and metabolic comorbidities. Dermatol Clin. 2015;33: $41-55$.

11. Mrowietz U, Kragballe K, Reich K, et al. Definition of treatment goals for moderate to severe psoriasis: a European consensus. Arch Dermatol Res. 2011;303:1-10.

12. Girolomoni G, Altomare G, Ayala F, et al. Differential management of mild-to-severe psoriasis with biologic drugs: an Italian Delphi consensus expert panel. J Dermatolog Treat. 2015;26:128-133.

13. Weger W. Current status and new developments in the treatment of psoriasis and psoriatic arthritis with biological agents. BrJ Pharmacol. 2010;160:810-820.

14. Dubois DS, Pouliot R. Promising new treatments for psoriasis. Scientific World J. 2013;2013:980419.

15. Gniadecki R, Kragballe K, Dam TN, Skov L. Comparison of drug survival rates for adalimumab, etanercept and infliximab in patients with psoriasis vulgaris. Br J Dermatol. 2011;164:1091-1096.
16. Yeung H, Wan J, Van Voorhees AS, et al. Patient-reported reasons for the discontinuation of commonly used treatments for moderate to severe psoriasis. J Am Acad Dermatol. 2013;68:64-72.

17. Di Lernia V, Ricci C, Lallas A, Ficarelli E. Clinical predictors of nonresponse to any tumor necrosis factor (TNF) blockers: a retrospective study. J Dermatolog Treat. 2014;25:73-74.

18. Di Lernia V, Tasin L, Pellicano R, et al. Impact of body mass index on retention rates of anti-TNF-alfa drugs in daily practice for psoriasis. $J$ Dermatolog Treat. 2012;23:404-409.

19. De Simone C, Amerio P, Amoruso G, et al. Immunogenicity of antiTNF $\alpha$ therapy in psoriasis: a clinical issue? Expert Opin Biol Ther. 2013;13:1673-1682.

20. Armstrong AW, Robertson AD, Wu J, Schupp C, Lebwohl MG. Undertreatment, treatment trends, and treatment dissatisfaction among patients with psoriasis and psoriatic arthritis in the United States: findings from the national psoriasis foundation surveys, 2003-2011. JAMA Dermatol. 2013;149:1180-1185.

21. Mansouri Y, Goldenberg G. New systemic therapies for psoriasis. Cutis. 2015;95:155-160.

22. Patterson H, Nibbs R, McInnes I, Siebert S. Protein kinase inhibitors in the treatment of inflammatory and autoimmune diseases. Clin Exp Immunol. 2014;176:1-10.

23. Murray PJ. The JAK-STAT signaling pathway: input and output integration. J Immunol. 2007;178:2623-2629.

24. Velazquez L, Fellous M, Stark GR, Pellegrini S. A protein tyrosine kinase in the interferon $\alpha / \beta$ signaling pathway. Cell. 1992;70:313-322.

25. Muller M, Briscoe J, Laxton C, et al. The protein tyrosine kinase JAK1 complements defects in interferon-alpha/beta and -gamma signal transduction. Nature. 1993;366:129-135.

26. Darnell JE Jr, Kerr IM, Stark GR. Jak-STAT pathways and transcriptional activation in response to IFNs and other extracellular signalling proteins. Science. 1994;264:1415-1421.

27. O'Shea JJ, Gadina M, Schreiber RD. Cytokine signaling in 2002: new surprises in the Jak/Stat pathway. Cell. 2002;109:121-131.

28. Leonard WJ, O'Shea JJ. Jaks and STATs: biological implications. Annu Rev Immunol. 1998;16:293-322.

29. Lindstrom TM, Robinson WH. A multitude of kinases - which are the best targets in treating rheumatoid arthritis? Rheum Dis Clin North Am. 2010;36:367-383.

30. Ghoreschi K, Gadina M. JAKpot! New small molecules in autoimmune and inflammatory. Exp Dermatol. 2014;23:7-11.

31. Borie DC, O'Shea JJ, Changelian PS. JAK3 inhibition, a viable new modality of immunosuppression for solid organ transplants. Trends Mol Med. 2004;10:532-541.

32. Macchi P, Villa A, Giliani S, et al. Mutations of Jak-3 gene in patients with autosomal severe combined immune deficiency (SCID). Nature. 1995; 377:65-68.

33. Chiricozzi A, Faleri S, Saraceno R, et al. Tofacitinib for the treatment of moderate-to-severe psoriasis. Expert Rev Clin Immunol. 2015; 11:443-455.

34. Kyttaris VC. Kinase inhibitors: a new class of antirheumatic drugs. Drug Des Devel Ther. 2012;6:245-250.

35. Chang BY, Zhao F, He X, et al. JAK3 inhibition significantly attenuates psoriasiform skin inflammation in CD18 mutant PL/J mice. J Immunol. 2009;183:2183-2192.

36. Ghoreschi K, Jesson MI, Li X, et al. Modulation of innate and adaptive immune responses by tofacitinib (CP-690, 550). J Immunol. 2011; 186:4234-4243.

37. Fridman JS, Scherle PA, Collins R, et al. Selective inhibition of JAK1 and JAK2 is efficacious in rodent models of arthritis: preclinical characterization of INCB028050. J Immunol. 2010;184:5298-5307.

38. Boy MG, Wang C, Wilkinson BE, et al. Double-blind, placebo-controlled, dose-escalation study to evaluate the pharmacologic effect of CP-690,550 in patients with psoriasis. $J$ Invest Dermatol. 2009;129:2299-2302.

39. Kubo S, Yamaoka K, Kondo M, et al. The JAK inhibitor, tofacitinib, reduces the $\mathrm{T}$ cell stimulatory capacity of human monocyte-derived dendritic cells. Ann Rheum Dis. 2014;73:2192-2198. 
40. Ghoreschi K, Laurence A, O'Shea JJ. Janus kinases in immune cell signalling. Immunol Rev. 2009;228:273-287.

41. Hsu L, Armstrong AW. JAK inhibitors: treatment efficacy and safety profile in patients with psoriasis. J Immunol Res. 2014;2014:283617.

42. Pesu M, Laurence A, Kishore N, Zwillich SH, Chan G, O'Shea JJ. Therapeutic targeting of Janus kinases. Immunol Rev. 2008;223:132-142.

43. Papp KA, Menter MA, Abe M, et al. Tofacitinib, an oral Janus kinase inhibitor, for the treatment of chronic plaque psoriasis: results from two randomized, placebo-controlled, phase III trials. Br J Dermatol. 2015; 173:949-961.

44. Bachelez H, van de Kerkhof PC, Strohal R, et al. Tofacitinib versus etanercept or placebo in moderate-to-severe chronic plaque psoriasis: a phase 3 randomised non-inferiority trial. Lancet. 2015;386: $552-561$.

45. Bissonnette R, Iversen L, Sofen $\mathrm{H}$, et al. Tofacitinib withdrawal and retreatment in moderate-to-severe chronic plaque psoriasis: a randomized controlled trial. Br J Dermatol. 2015;172:1395-1406.

46. Meyer DM, Jesson MI, Li X, et al. Anti-inflammatory activity and neutrophil reductions mediated by the JAK1/JAK3 inhibitor, CP-690,550, in rat adjuvant-induced arthritis. $J$ Inflamm (Lond). 2010;7:41.

47. Winthrop KL, Park SH, Gul A, et al. Tuberculosis and other opportunistic infections in tofacitinib-treated patients with rheumatoid arthritis. Ann Rheum Dis. Epub 2015 Aug 28. doi:10.1136/annrheumdis-2015207319.

48. Wollenhaupt J, Silverfield J, Lee EB, et al. Safety and efficacy of tofacitinib, an oral janus kinase inhibitor, for the treatment of rheumatoid arthritis in open-label, long term extension studies. J Rheumatol. 2014;41:837-852.

49. Strand V, Ahadieh S, French J, et al. Systematic review and metaanalysis of serious infections with tofacitinib and biologic diseasemodifying antirheumatic drug treatment in rheumatoid arthritis clinical trials. Arthritis Res Ther. 2015;17:362.

50. XELJANZ ${ }^{\circledR}$ (tofacitinib) tablets [prescribing information]. New York: Pfizer Labs. 2012. Available from:http://www.accessdata.fda.gov/ drugsatfda_docs/label/2012/203214s0001bl.pdf. Accessed 19 January, 2016 .
51. Gupta P, Alvey C, Wang R, et al. Lack of effect of tofacitinib (CP-690,550) on the pharmacokinetics of the CYP3A4 substrate midazolam in healthy volunteers: confirmation of in vitro data. $\mathrm{Br} \mathrm{J} \mathrm{Clin}$ Pharmacol. 2012;74:109-115.

52. Ports WC, Khan S, Lan S, et al. A randomized phase 2a efficacy and safety trial of the topical Janus kinase inhibitor tofacitinib in the treatment of chronic plaque psoriasis. Br J Dermatol. 2013;169:137-145.

53. Ports WC, Feldman SR, Gupta P, et al. Randomized pilot clinical trial of tofacitinib solution for plaque psoriasis: challenges of the intra-subject study design. J Drugs Dermatol. 2015;14:777-784.

54. Lee EB, Fleischmann R, Hall S, et al. Tofacitinib versus methotrexate in rheumatoid arthritis. N Engl J Med. 2014;370:2377-2386.

55. van Vollenhoven RF, Fleischmann R, Cohen S, et al. Tofacitinib or adalimumab versus placebo in rheumatoid arthritis. NEngl J Med. 2012;367: 508-519.

56. Vivar N, Van Vollenhoven RF. Advances in the treatment of rheumatoid arthritis. F1000Prime Rep. 2014;6:31.

57. Brittany G, Craiglow, King BA. Killing two birds with one stone: oral tofacitinib reverses alopecia universalis in a patient with plaque psoriasis. J Invest Dermatol. 2014;134:2988-2990.

58. Craiglow BG, King BA. Tofacitinib citrate for the treatment of vitiligo: a pathogenesis-directed therapy. JAMA Dermatol. 2015;151:1110-1112.

59. Xing L, Dai Z, Jabbari A, et al. Alopecia areata is driven by cytotoxic T lymphocytes and is reversed by JAK inhibition. Nat Med. 2014;20: 1043-1049.

60. Harel S, Higgins CA, Cerise JE, et al. Pharmacologic inhibition of JAKSTAT signaling promotes hair growth. Sci Adv. 2015;23;1:e1500973.

61. Rashighi M, Agarwal P, Richmond JM, et al. CXCL10 is critical for the progression and maintenance of depigmentation in a mouse model of vitiligo. Sci Transl Med. 2014;6:223.

62. Levy LL, Urban J, King BA. Treatment of recalcitrant atopic dermatitis with the oral Janus kinase inhibitor tofacitinib citrate. J Am Acad Dermatol. 2015;73:395-399.
Drug Design, Development and Therapy

\section{Publish your work in this journal}

Drug Design, Development and Therapy is an international, peerreviewed open-access journal that spans the spectrum of drug design and development through to clinical applications. Clinical outcomes, patient safety, and programs for the development and effective, safe, and sustained use of medicines are a feature of the journal, which

\section{Dovepress}

has also been accepted for indexing on PubMed Central. The manuscript management system is completely online and includes a very quick and fair peer-review system, which is all easy to use. Visit http://www.dovepress.com/testimonials.php to read real quotes from published authors. 